\title{
EXPLORING THE ROLE OF SHEAR STRESS AND SEVERE TURBULENCE IN DOWNSTREAM FISH PASSAGE
}

Abstract

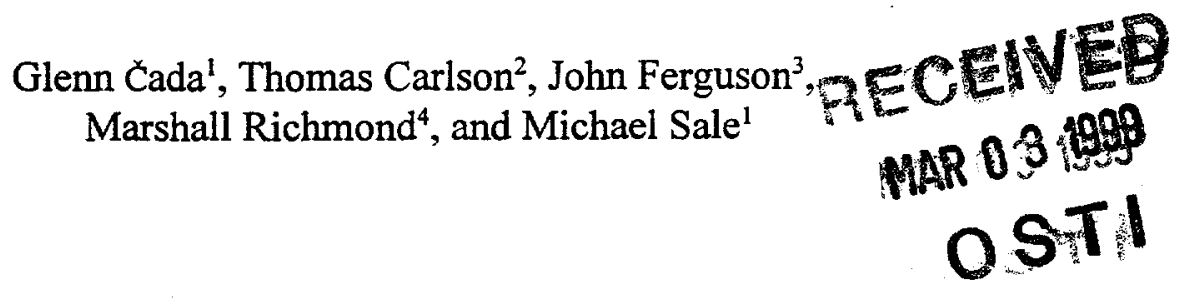

Fish may be exposed to damaging levels of fluid shear stress and turbulence while passing through hydroelectric power plants. The generally assumed locations for such potential damage are the turbine and draft tube passages, although it is possible that fish are also injured during passage over the spillway or through sluiceways and fish bypass outfalls. Unless mitigated, fluid-induced injuries and mortality could frustrate efforts to develop advanced, "fish-friendly" turbines or to provide safe alternate downstream passages. The effects of shear stress and turbulence on fish are poorly understood, in part because of the difficulties in conceptualizing these phenomena, determining their magnitudes and distribution within hydroelectric systems, and then recreating them in a controlled laboratory environment.

We define the fluid phenomena that are relevant to the assessment of effects on fish. The magnitudes of fluid stresses associated with man-altered aquatic environments are often considerably higher than those found in natural environments (e.g., normal river flows). However, levels of shear stresses that occur during flash floods appear to be comparable to those expected within a turbine. Past studies of the effects of shear stress on fish are of limited value, mainly because of their narrow scope and lack of instrumentation to measure velocities on appropriately small scales. A laboratory experiment to study the effects of shear stress and turbulence on fish is described.

1 Environmental Sciences Division, Oak Ridge National Laboratory, Oak Ridge, TN 37831-6036

2 U.S. Army Corps of Engineers, CENPP-PE-E, P.O. Box 2946, Portland, OR 97208-2946

3 National Marine Fisheries Service, 2725 Montlake Boulevard, East, Seattle, WA 98112

4 Pacific Northwest National Laboratory, Battelle Boulevard, P.O. Box 999, Richland, WA 99352 


\section{DISCLAIMER}

This report was prepared as an account of work sponsored by an agency of the United States Government. Neither the United States Government nor any agency thereof, nor any of their employees, make any warranty, express or implied, or assumes any legal liability or responsibility for the accuracy, completeness, or usefulness of any information, apparatus, product, or process disclosed, or represents that its use would not infringe privately owned rights. Reference herein to any specific commercial product, process, or service by trade name, trademark, manufacturer, or otherwise does not necessarily constitute or imply its endorsement, recommendation, or favoring by the United States Government or any agency thereof. The views and opinions of authors expressed herein do not necessarily state or reflect those of the United States Government or any agency thereof. 


\section{DISCLAIMER}

Portions of this document may be illegible in electronic image products. Images are produced from the best available original document. 


\section{Introduction}

Fluid shear and turbulence are natural phenomena that are important to fish. Aquatic organisms have developed numerous adaptations to the levels of shear and turbulence found in normal river flows (Vogel 1981). In fact, proper reaction to turbulence may promote movement among migratory fish (Coutant 1998) or prevent displacement of resident fish (Shtaf et al. 1983; Skorobogatov and Pavlov 1991).

However, elevated levels of shear and turbulence may be damaging. These can occur in both natural and man-made environments. For example, concerns have been expressed by numerous investigators that excessive fluid stresses associated with passage through hydroelectric turbines, spillways, and fish bypass systems may have adverse effects on downstream migrating fish (Čada 1997). Unfortunately, the effects of shear and turbulence on turbine-passed fish are poorly understood, in no small part because of the difficulties in understanding these flow phenomena, determining their magnitudes and distribution within hydroelectric turbine systems, and then recreating them in a controlled laboratory environment. In this paper, we define the terms associated with these fluid phenomena and compare values reported in the literature. We outline an ongoing laboratory experiment that exposes fish to shear and turbulence values likely to be experienced at hydroelectric power plants.

\section{Definition of Terms}

Shear and turbulence are both expressions of changes in water velocity. Velocity (v) is measured in distance (y) per unit time, e.g., $\mathrm{m} / \mathrm{s}$. Water velocity is important for transporting organisms or their food and for creating aquatic habitats. Uniform velocities in a stream or artificial conduit do not harm fish; a fish moving $0.3 \mathrm{~m} / \mathrm{s}$ or 30 $\mathrm{m} / \mathrm{s}$ will not be injured or disoriented if velocities of the water mass in which the fish is traveling are relatively uniform. However, when water velocities change on scales comparable to the size of a fish, damaging shear stresses and turbulence can occur.

Shear occurs when two water masses of different velocities intersect or are adjacent to each other. Shear is defined as the change in water velocity (v) over distance (y), expressed as $\mathrm{m} / \mathrm{s}$ per $\mathrm{m}$, or units/s.

$$
\text { shear }=\mathrm{dv} / \mathrm{dy}
$$

For example, if velocities of two adjacent water masses differed by $3 \mathrm{~m} / \mathrm{s}$ over a distance of $0.1 \mathrm{~m}$, the resultant shear would be $30 / \mathrm{s}$. If there was no resistance to flow (no viscosity), the fish would just spin freely in response to the different velocities impinging on it and would not experience any harm (other than dizziness). However, because the water in which the fish is traveling has viscosity, force is exerted on the fish as a result of encountering a mass of water moving at a different velocity. Depending on its magnitude, this force may be harmful or inconsequential. Shear is not a sufficient 
expression of potential damage to fish - estimates of shear must be combined with estimates of viscosity to obtain shear stress.

Force is defined as mass multiplied by acceleration and is expressed in Newtons (N). For a fish striking a wall, the force would be the mass of the fish multiplied by the deceleration. A large fish rapidly decelerating from $10 \mathrm{~m} / \mathrm{s}$ to $0 \mathrm{~m} / \mathrm{s}$ would experience more force hitting the wall than a small fish moving in the low-velocity boundary layer along the wall. This same relationship is true if, instead of a wall, the fish encounters another water mass of different velocity. Important factors that govern the effect of force are (1) size of the fish (larvae have lower mass than adults so they strike the wall/water mass with less force), (2) life stage (larvae are more sensitive to a given amount of force than adults), (3) the way in which the fish strikes the wall or water mass (entire side of the body vs. head-on). This last factor leads to a consideration of pressure. Pressure is force per unit area, applied perpendicular to the body surface, expressed in $\mathrm{N} / \mathrm{m}^{2}$. If all the force (fish's mass multiplied by acceleration) of striking the wall is focused on one small point (eyeball), there will be much greater pressure and injury than if the entire side of the fish's body strikes the wall. This same relationship is true if, instead of a wall, the fish strikes another water mass of different velocity. The force associated with the different water mass could be distributed relatively uniformly over the whole body, or it could be experienced as a pin-point jet. The location and amount of the fish's body upon which the forces are focused have a bearing on the resultant damage.

Shear stress, like pressure, is force per unit area. In laminar flow, it is estimated by Newton's equation:

$$
\tau_{\text {laminar }}=\mu(\mathrm{dv} / \mathrm{dy})
$$

where $\mu$ is dynamic (laminar) viscosity. Values of $\mu$ for different fluids at different temperatures may be obtained from tables.

The difference between pressure and shear stress is the direction in which the force is applied. In pressure the force acts perpendicular to the surface, whereas a shear force acts parallel to it (Gordon et al. 1992). Shear stress has the same units as pressure, $\mathrm{N} / \mathrm{m}^{2}$. Shear stresses can be experienced by a fish passing between two water masses of different velocities, or when a fish slides along a solid structure such as a wall or turbine blade (commonly termed abrasion). Damage from passage near a structure may occur from very high shear stresses within the liquid boundary layer, even without physical contact with the solid surface. The same considerations associated with pressure also apply to shear stress, i.e., a given amount of shear force is expected to be more damaging if applied to a small area of the body than a large area.

Because of high water velocities and edge effects of structures, flows in the turbine will be turbulent, rather than laminar. Turbulent flow occurs when fluid 
particles move in a highly irregular manner, even if the fluid as a whole is traveling in a single direction. That is, there are intense, small-scale motions present in directions other than that of the main, large-scale flow (Vogel 1981). These small-scale motions are often in the form of vortices. Unlike laminar flow, which is most easily described by a linear equation, turbulent flow can only be defined statistically (Gordon et al. 1992); descriptions of the overall motion within turbulent flows cannot be taken as describing the paths of individual particles (Vogel 1981). Turbulence exists at all scales in nature, from the swirling motion created when a salmon scoops out a redd (scales smaller than the size of the fish) to large pulses of flow in a river (scales much larger than a fish). Similarly, within a hydroelectric turbine, turbulence occurs at different scales. Smaller-scale turbulence, which occurs throughout turbine passage, can distort and compress portions of the fish's body. Larger-scale turbulence may be most pronounced in the draft tube, where water flow is decelerating, expanding into a larger passage, and has a swirl imparted on it by the turbine runner. Fixed structures in the draft tube (walls and support piers) may cause secondary flows, i.e., flows moving in opposite directions from the main flow moving out of the draft tube and into the tailrace. These chaotic flow conditions (small-scale turbulence, larger-scale flow pulses, vortices, and secondary flows) will distort and spin the fish, and at the least may cause disorientation. It has been suggested that this turbulence-caused disorientation, while perhaps not injuring the fish directly, may leave turbine-passed fish more susceptible to predators in the tailrace.

Shear stress can also be estimated for turbulent flows, but it is more complicated. For turbulent flows, Eq. (2) is modified to incorporate another term, eddy viscosity (€):

$$
\tau_{\text {turbulent }}=(\mu+\epsilon)(\mathrm{dv} / \mathrm{dy})
$$

Unlike $\mu$, there are no tables that give values for $\epsilon$. Eddy viscosity is dependent on how vigorous the turbulence is, and must be found by experimentation. However, because $\epsilon$ is a function of the first and second derivatives of velocity (i.e., dv/dy and $\left.\mathrm{d}^{2} \mathrm{v} / \mathrm{dy}^{2}\right)$, it can be calculated from the velocity profile. If the flow is entirely laminar, $\epsilon$ is zero and Eq. 3 reduces to Eq. 2. For fully turbulent flow, effects due to dynamic viscosity are negligible $(\mu<\epsilon)$, and Eq. 3 reduces to:

$$
\tau_{\text {turbulent }}=\epsilon(\mathrm{dv} / \mathrm{dy})
$$

Turbulence can also be described by a measure called turbulence intensity (Gordon et al. 1992). If we think of local velocity in a turbulent region as composed of a mean value plus a component which represents fluctuation about the mean, turbulence intensity is a measure of the magnitude of the turbulent fluctuations about the mean. Greater fluctuations from the mean flow equals greater turbulence. If a series of instantaneous velocity measurements are made at a point, the turbulence intensity at that point can be expressed as the root mean square of these measured values. 
Shear Stress in the Environment

Table 1 displays values for shear stress that have been reported for a variety of natural and man-altered aquatic environments. Not surprisingly, estimated values of shear stress in streams and rivers under normal flows are generally low, most often less than $100 \mathrm{~N} / \mathrm{m}^{2}$. Because this is the natural environment for fish, it can be expected that these values would not be damaging. On the other hand, shear stress estimates for flash floods in small river basins may be several hundred to as high as $2,600 \mathrm{~N} / \mathrm{m}^{2}$. Fish are often displaced downstream and killed in flash floods, although shear stress may not be the sole cause.

\section{Shear and Shear Stress at Hydroelectric Power Plants}

The largest values of shear in a turbine water passage are likely to occur in the narrow boundary layer near structures and in association with vortices: near the periphery of the blade (blade tip vortex), near the hub (hub leakage vortex), and in the center of a Francis turbine (rope vortex). We have not found estimates of the magnitude of shear near such vortices. It has been suggested that shear values may be $400-600 / \mathrm{s}$ in a narrow boundary layer near structures such as the wicket gate, stay vane, and runner blade leading edge (Voith Hydro, Inc. 1997). However, the bulk of the flow through a Kaplan turbine may create much smaller shears, perhaps in the neighborhood of 50/s.

\begin{tabular}{||l|c|}
\hline \multicolumn{2}{|c|}{ Table 1. Published estimates of shear stress $\left(\mathbf{N} / \mathbf{m}^{2}\right)$ in natural and } \\
man-altered aquatic environments. & Shear stress $\left.\mathbf{~} \mathbf{N} / \mathbf{m}^{2}\right)$ \\
\hline Watér column in a trout stream, average flow & $<1.0$ \\
\hline Small streams, near bed & $<1-7$ \\
\hline $\begin{array}{l}\text { Medium-sized streams, near bed (90 } \\
\text { measurements) }\end{array}$ & most $<30$, but some $>200$ \\
\hline Flash floods, small basins & $61-2600$ \\
\hline Floods, large rivers & $6-10$ \\
\hline Bulb turbine draft tube & $500-5,421$ \\
\hline Near ships' hulls and wakes & $7.6-40.4$ \\
\hline Near barge propeller & $\geq 5,000$ \\
\hline Spillways & $?$ \\
\hline
\end{tabular}


Estimates of shear stresses in the draft tube of a bulb turbine and near a ship's propeller may exceed $5,000 \mathrm{~N} / \mathrm{m}^{2}$ (Table 1). It should be remembered that these large values may occur in only a small portion of the turbine passage. For example, McEwen and Scobie (1992) estimated that shear stress values in a bulb turbine would be less than $500 \mathrm{~N} / \mathrm{m}^{2}$ in about 85 percent of the draft tube and less than $1,000 \mathrm{~N} / \mathrm{m}^{2}$ in over 90 percent of the draft tube (Table 2). Shear stresses between 4,000 and $5,000 \mathrm{~N} / \mathrm{m}^{2}$ would occur in only about 2 percent of the passage.

Water discharged from dams via spillways can reach velocities of tens of meters per second, which can create high values of shear and shear stress, especially near solid surfaces. At high velocities (12 to $15 \mathrm{~m} / \mathrm{s}$ or more), surface irregularities in the dam can cause flow separation that is sufficient to lower water pressure below the local vapor pressure and cause cavitation (Chanson 1989). Flow separations that can cause cavitation might also be expected to exert damaging shear stress on fish being transported in these spill flows. The effects of spillway discharges on fish have not been examined mechanistically (R2 1998).

\begin{tabular}{|c|c|c|}
\hline $\begin{array}{c}\text { Shear Stress } \\
\left(\mathbf{N} / \mathbf{m}^{2}\right)\end{array}$ & $\begin{array}{c}\text { Off-design } \\
\text { operation (\%) }\end{array}$ & $\begin{array}{c}\text { Design } \\
\text { operation (\%) }\end{array}$ \\
\hline $50-100$ & 56.89 & 52.10 \\
\hline $100-500$ & 28.14 & 31.14 \\
\hline $500-1000$ & 6.21 & 9.21 \\
\hline $1000-2000$ & 2.99 & 3.89 \\
\hline $2000-3000$ & 2.40 & 2.10 \\
\hline $3000-4000$ & 1.20 & 1.50 \\
\hline $4000-5000$ & 2.10 & 0.00 \\
\hline
\end{tabular}

\section{Description of U.S. Department of Energy (DOE) Experiments}

The Advanced Hydropower Turbine System (AHTS) Program of the U.S. DOE has begun experiments aimed at determining whether the shear stresses and turbulence that occur within hydroelectric turbines are injurious to fish. The basic elements of the experimental procedure are these: 
1. Shear and turbulence are generated in a large, water-filled flume by means of a water jet created by a submerged nozzle.

2. When flow in the flume downstream from the jet has "stabilized," Laser Doppler Velocimetry (LDV) is used to characterize the shear zone velocity profiles in the $\mathrm{x}, \mathrm{y}$, and $\mathrm{z}$ dimensions.

3. An individual fish is placed directly into the shear zone through a release tube at a predetermined angle, location, and orientation and is swept downstream by the flow of water.

4. High-speed video photography is used to record the three-dimensional path of each fish.

5. The path of each fish is superimposed on the previously determined velocity profile in order to estimate (a) the velocity changes experienced by the fish and (b) the region and areal extent of the fish's body exposed to shear stress and turbulence.

6. Hydraulic conditions (shear, shear stress, turbulence, vorticity) experienced by each fish are estimated.

7. The estimates of shear stress are plotted against some measure of biological response (e.g., mortality, external creases, torn operculum, percent descaling, disorientation).

As the fish strike the shear zone created by the jet, they encounter a water mass moving at much higher velocities, so the shear and shear stress increase rapidly. Subsequently, as the fish are swept down the flume and the high-velocity water mass spreads out and slows, the velocity differentials are smaller and the shear stresses experienced by the fish decrease with travel time (or distance) from the nozzle. Because each fish will follow a different path through the flume, each fish will be exposed to a different velocity regime and for a different exposure time.

The independent variable (dose) in these experiments is a function of turbulent shear stress $[(\mu+\epsilon)(d v / d y)]$, exposure time $(t)$, turbulence intensity, and the surface area of the fish exposed to the shear stress and turbulence. These are estimated in the experimental flume with the use of LDV and high speed video photography and are used in a multivariate regression model for comparison to some biological response.

Many of the parameters considered in these experiments (shear, turbulence, and exposure time) are factors that can be altered by turbine designers as they attempt to reduce each of the possible injury mechanisms (e.g., strike, pressure, shear) to acceptable levels. In addition, these data may also help us understand whether other downstream fish passage routes (spillways, sluiceway outfalls, bypass outfalls) and other situations in which fish may be exposed to shear (e.g., proximity to vessel hulls and propellers) are damaging. 


\section{Acknowledgements}

We thank the Technical Committee of the U.S. DOE's Advanced Hydropower Turbine Systems Program for their advice, and Chuck Coutant and Steve Bao for their reviews of this paper. The research was sponsored by the Office of Geothermal Technologies, U.S. Department of Energy, under contract number DE-AC05960R22464 with Lockheed Martin Energy Research Corp. This is Publication No. 4856, Environmental Sciences Division, ORNL.

\section{References}

Cada, G.F. 1997. Shaken, not stirred: the recipe for a fish-friendly turbine. p. 374-382 IN Waterpower '97. Proceedings of the International Conference on Hydropower. D.J. Mahoney (ed.). American Society of Civil Engineers, New York, NY. 2267 p.

Chanson, H. 1989. Flow downstream of an aerator - aerator spacing. Journal of Hydraulic Research 27(4):519-536.

Coutant, C.C. 1998. Turbulent attraction flows for juvenile salmonid passage at dams. ORNL/TM-13608. Oak Ridge National Laboratory, Oak Ridge, TN. 28 p.

Gordon, N.D., T.A. McMahon, and B.L. Finlayson. 1992. Stream hydrology: An introduction for ecologists. John Wiley \& Sons, New York, NY. 526 p.

McEwen, D. and G. Scobie. 1992. Estimation of the hydraulic conditions relating to fish passage through turbines. NPC001. National Engineering Laboratory, East Kilbride, Glasgow. 155 p.

R2 (R2 Resource Consultants, Inc.). 1998. Annotated bibliography of literature regarding mechanical injury with emphasis on effects from spillways and stilling basins. Final Report prepared for U.S. Army Corps of Engineers, Portland District, Portland, OR.

Shtaf, L.G., D.S. Pavlov, M.A. Skorobogatov, and A. S. Barekyan. 1983. The influence of flow turbulence on fish behavior. Journal of Ichthyology 23(2):129-140.

Skorobogatov, M.A. and S.D. Pavlov. 1991. A study of the orientation of young roach, Rutilus rutilus, with respect to current velocity. Journal of Ichthyology 31:142148.

Vogel, S. 1981. Life in moving fluids: The physical biology of flow. Princeton University Press. Princeton, New Jersey. 352 p. 
Voith Hydro, Inc. 1997. Development of environmentally advanced hydropower turbine system design concepts. Report No. 2677-0141 to the U.S. Department of Energy, Idaho Operations Office and Hydropower Research Foundation, Inc.

"The submitted manuscript has been authored by a contractor of the U.S. Government under contract No. DE-AC05-960R22464. Accordingly, the U.S. Government retains a non-exclusive, royalty-free license to publish or reproduce the published form

of this contribution, or allow others to do so, for U.S. Government purposes." 